\title{
The current situation and challenges of screening for and treating hepatitis B in sub-Saharan Africa
}

\section{Charles Béguelin, Fatou Fall, Moussa Seydi \& Gilles Wandeler}

To cite this article: Charles Béguelin, Fatou Fall, Moussa Seydi \& Gilles Wandeler (2018): The current situation and challenges of screening for and treating hepatitis $B$ in sub-Saharan Africa, Expert Review of Gastroenterology \& Hepatology, DOI: 10.1080/17474124.2018.1474097

To link to this article: https://doi.org/10.1080/17474124.2018.1474097

Accepted author version posted online: 08 May 2018.

Submit your article to this journal $๘$

Џ Article views: 2

Q View related articles $₫$

View Crossmark data $\asymp$ 
Publisher: Taylor \& Francis

Journal: Expert Review of Gastroenterology \& Hepatology

DOI: $10.1080 / 17474124.2018 .1474097$

\section{Review}

\section{The current situation and challenges of screening for and treating hepatitis B in sub-Saharan Africa}

Charles Béguelin ${ }^{1}$, Fatou Fall ${ }^{2}$, Moussa Seydi ${ }^{3}$, Gilles Wandeler ${ }^{1,3,4}$

${ }^{1}$ Department of Infectious Diseases, Bern University Hospital, University of Bern, Switzerland ${ }^{2}$ Division of Gastroenterology and Hepatology, Hôpital Principal, Dakar, Senegal, ${ }^{3}$ Department of Infectious Diseases, Hôpital Fann, Dakar, Senegal

${ }^{4}$ Institute of Social and Preventive Medicine, University of Bern, Switzerland

\section{Correspondence:}

Gilles Wandeler

Department of Infectious Diseases, Bern University Hospital, University of Bern Haus 5 - Inselspital, 3010 Bern, Switzerland

Email: gilles.wandeler@insel.ch

Telephone: +41316322525 


\begin{abstract}
Introduction: Hepatitis B virus (HBV) infection is the most important cause of hepatocellular carcinoma in sub-Saharan Africa (SSA). Although the tools to curb the epidemic are known, only a minority of HBV-infected persons are currently diagnosed and treated.
\end{abstract}

Areas covered: We discuss HBV epidemiological trends in SSA, describe important determinants of its natural history, and summarize current knowledge on the continuum of HBV care. Using the results of a systematic review of the literature, we describe the proportion of patients with liver fibrosis at presentation for care. Throughout the manuscript, we highlight major research gaps and explore potential ways to improve uptake of HBV testing, evaluation of liver disease, access to antiviral therapy and monitoring of complications.

Expert commentary: Less than $1 \%$ of HBV-infected individuals are diagnosed in SSA, despite the availability of rapid tests with good diagnostic accuracy. Up to $15 \%$ of individuals enter care with liver cirrhosis, a clear indication for antiviral therapy. Although the proportion of patients eligible for immediate antiviral treatment is generally below $20 \%$, there are few published data from prospective cohort studies. The incidence of hepatocellular carcinoma could be reduced with improved access to antiviral therapy.

Key words: hepatitis B virus; sub-Saharan Africa; liver cirrhosis, hepatocellular carcinoma, antiviral therapy 


\section{Introduction}

Worldwide, hepatitis B virus (HBV) infection causes approximately 900,000 deaths per year and is the most important single risk factor for hepatocellular carcinoma (HCC) ${ }^{1}$. Whereas $3.6 \%$ of the global population is affected by chronic HBV infection, defined as a single positive HBV surface antigen (HBsAg) by the World Health Organization (WHO $)^{2}$, most countries in sub-Saharan Africa (SSA) have a prevalence above $8 \%{ }^{2}$. In 2016, the World Health Assembly endorsed the Global Health Sector Strategy on viral hepatitis, calling for its elimination as a public health threat by $2030^{3}$. In order to achieve this ambitious goal, the World Health Organization (WHO) estimates that new infections need to be reduced by $90 \%$ and hepatitis-related mortality by $65 \%$. To guide the road to elimination and inform national hepatitis strategies, $\mathrm{WHO}$ has issued comprehensive guidelines for testing and management of HBV infection ${ }^{4,5}$. Furthermore, a list of specific objectives to be achieved by 2030 was established: $90 \%$ of infected persons need to be diagnosed, and $80 \%$ of eligible individuals treated $^{3}$.

In SSA, each step of the HBV continuum of care is severely affected by the lack of resources, awareness and political will (Figure 1). For instance, only $0.3 \%$ of the HBVinfected persons have been diagnosed to date, despite the availability of cheap, highly sensitive HBsAg tests. Similar observations can be made about uptake of HBV treatment: the current standard of care for HBV therapy, tenofovir disoproxil fumarate (TDF), suppresses HBV replication in nearly all patients, is well-tolerated, and now increasingly available as a low-cost generic drugs. Yet, the proportion of HBV-infected individuals in need of antiviral therapy in SSA remains largely unknown, and access to treatment very limited. In addition, training of health-care workers on HBV management is insufficient: for example, in a recent survey conducted among a representative sample of physicians in Senegal, only $51 \%$ of them knew that HBV vaccination was safe for newborns ${ }^{6}$.

Generally, epidemiological and clinical research on HBV infection in SSA has been developing slowly and has so far not been able to attract appropriate funding. In this article, we review recent evidence on the determinants of the main components of the continuum of $\mathrm{HBV}$ care in SSA, from HBV testing to staging of liver disease, treatment and monitoring (Figure 1). We highlight the main scientific knowledge gaps and important challenges for the implementation of management strategies in order to guide further efforts in the field. Although the prevention of mother-to-child transmission and vaccination are cornerstones of the global strategy to eliminate HBV infection, we do not discuss HBV prevention aspects in this manuscript, but instead focus on the management of HBV-infected populations in SSA. 
Figure 1: The key components of HBV care and related research gaps and challenges in sub-Saharan Africa

(HBsAg: hepatitis B surface antigen; NIT: non-invasive tests; TE: transient elastography; HCC: hepatocellular carcinoma)

\begin{tabular}{|c|c|c|c|}
\hline & Key components & Knowledge gaps & Implementation challenges \\
\hline Testing & $\begin{array}{l}\text { - Routine screening for } \mathrm{HBsAg} \\
\text { - Testing for coinfections }\end{array}$ & $\begin{array}{l}\text { Risk groups, Test validation } \\
\text { Prevalence, risk groups }\end{array}$ & $\begin{array}{l}\text { Prioritization of specific populations } \\
\text { Availability and cost of diagnostic tests }\end{array}$ \\
\hline Staging & $\begin{array}{l}\text { - Measurement of transaminases } \\
\text { - Measurement of HBV viral load } \\
\text { - Liver fibrosis assessment }\end{array}$ & $\begin{array}{l}\text { Need for serial measurements } \\
\text { Correlation with other markers } \\
\text { Validity of NIT, prevalence of cirrhosis }\end{array}$ & $\begin{array}{l}\text { Retention in care } \\
\text { Cost and laboratory capacity } \\
\text { Cost, training and availability of TE }\end{array}$ \\
\hline atment & $\begin{array}{l}\text { - Eligibility assessment } \\
\text { - Treatment initiation and adherence }\end{array}$ & $\begin{array}{l}\text { Who to treat, when to stop } \\
\text { Impact of adherence on outcomes }\end{array}$ & $\begin{array}{l}\text { Retention in care } \\
\text { Securing treatment adherence }\end{array}$ \\
\hline lonitoring & $\begin{array}{l}\text { - Treatment outcome } \\
\text { - HCC screening }\end{array}$ & $\begin{array}{l}\text { Frequency of virological monitoring } \\
\text { Who to screen }\end{array}$ & $\begin{array}{l}\text { Retention, Cost } \\
\text { Availability of ultrasound and training }\end{array}$ \\
\hline
\end{tabular}

\section{Epidemiological trends and natural history of HBV infection in Africa}

\subsection{Epidemiology}

Approximately 60 million people live with a chronic HBV infection in Africa (estimated prevalence: $6.1 \%, 95 \%$ confidence interval $[\mathrm{Cl}]$ 4.6-8.5) ${ }^{3}$. A recent systematic review of the global prevalence of HBV infection showed high estimates across SSA: chronic HBV infection is hyper-endemic ( $>8 \%$ HBsAg-positivity in the general population) in parts of SSA, including most countries in West and Central Africa (Figure 2). Other countries, mainly in southern and Eastern Africa, are considered areas of intermediate endemicity (2\%-8\%), while Egypt and Morocco have a low endemicity level $(<2 \%)^{2}$. Most HBV-infected persons were born before $\mathrm{HBV}$ vaccine was available and widely used: although $\mathrm{HBV}$ vaccination is available since 1982, HBV immunization programs started only in the early 2000s in most of the African countries ${ }^{7}$. Worldwide, the estimated prevalence of chronic HBV infection among children under five years was $1.3 \%$ in 2015 , compared with $4.7 \%$ in the pre-vaccine era. However, the prevalence in young children remains above $3 \%$ in SSA, where the implementation of the birth-dose vaccine, a fundamental part of prevention of mother-to-child HBV transmission, has been very limited to date. 
Whereas early studies suggested a high variation in HBV prevalence estimates between countries and sub-groups of the population in SSA, these findings can often be explained by methodological differences ${ }^{7-10}$. A recent meta-analysis of 44 studies in Cameroon highlighted the stability of HBV prevalence across different sub-populations, despite a certain degree of heterogeneity between studies. The overall pooled seroprevalence was $11.2 \%$ (95\% Cl 9.7$12.8 \%$ ), and the estimate remained above $10 \%$ after the exclusion of studies in populations previously considered as high-risk $(10.6 \%, 95 \% \mathrm{Cl} 8.6-12.6 \%)^{11}$. In line with these findings, recent data from Senegal showed similar HBV prevalence estimates across different populations, including blood donors (17\%), prison inmates (14\%), military staff $(14 \%)$ and HIV-infected persons $(12 \%){ }^{12-15}$. Considering the consistent results from recent studies across SSA, WHO now recommends HBsAg testing at least once in the general population of this region.

Figure 2: Prevalence of chronic HBV infection in sub-Saharan Africa (adapted from Schweitzer et al. ${ }^{2}$ )

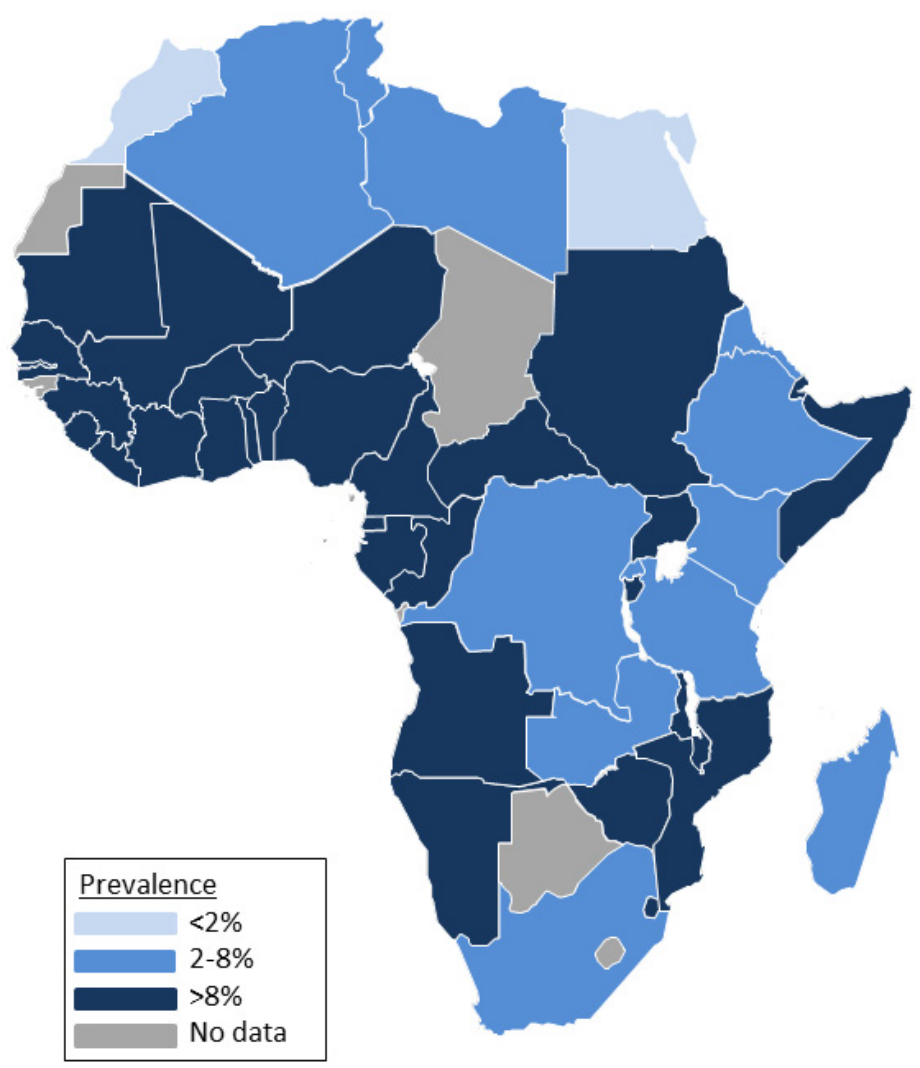

An estimated 2.7 million (interquartile range 1.8-3.9) of the 36.7 million persons living with HIV are co-infected with HBV, of whom $71 \%$ live in $\mathrm{SSA}^{16}$. HIV infection accelerates the progression of HBV-related liver disease and increases liver-related mortality ${ }^{17}$. In contrast to the situation in high-income countries, most persons acquire HBV during early childhood in 
SSA, long before being infected with HIV. Therefore, HBV prevalence among HIV-infected individuals is generally similar to estimates from the general population ${ }^{18}$. In the past ten years, funding for research in SSA has been widely driven by efforts to fight HIVIAIDS, which explains the extensive body of literature on HBV determinants in HIV-infected populations, as opposed to the general population. Although many epidemiological parameters are comparable, considerations about the natural history and treatment outcomes of HIV/HBVcoinfected persons can generally not be transferred to HIV-uninfected populations.

\subsection{HBV transmission and natural history}

In regions of low HBV endemicity such as Western Europe and North America, most infections occur in adolescents and young adults, mainly through sexual or percutaneous transmission ${ }^{19}$. By contrast, in SSA and Southeast Asia, the majority of HBV infections are acquired before the age of five. Perinatal transmission, which is most likely when HBVinfected mothers have a high HBV viral load (VL) and a positive hepatitis B envelope antigen $(\mathrm{HBeAg})$, predominates in Southeast Asia, whereas in SSA, children acquire HBV infection mainly through horizontal transmission from household contacts ${ }^{20,21}$. The proportion of $\mathrm{HBeAg}$-positive pregnant women in SSA is generally lower than in Asia, varying between $1 \%$ in Ghana and 24\% in Tanzania among HBV-monoinfected populations ${ }^{22-24}$. However, the risk of perinatal transmission seems to be higher among HIV/HBV-coinfected mothers, who are twice as likely to be HBeAg-positive and three times more likely to have a high HBV VL compared to HIV-uninfected ones ${ }^{25}$. Sharing of cutting objects, such a razors, as well as other percutaneous injuries, including circumcision and scarification, are thought to account for the majority of HBV transmissions in $\mathrm{SSA}^{26}$. Even though sexual transmission of HBV infection does not seem to be of major importance in SSA, several reports have highlighted the potential for acquiring HBV through this route. Studies from Somalia and Ethiopia reported a higher HBV prevalence among adults compared to children, suggesting infections through sexual intercourse ${ }^{27,28}$.

Several specificities of the natural history of HBV infection in SSA may have an impact on transmission, burden of disease and treatment outcomes. The risk for HBV infection of becoming chronic is inversely related to the age at infection. Ninety percent of those infected in the neonatal period develop chronic infection, whereas this is only the case for $20-50 \%$ of those infected during early childhood ( $<5$ years), and for $5 \%$ of adults. Compared to Asian populations, HBV-infected individuals in SSA are more likely to become HBeAg-negative early in the course of disease (Figure 3): the yearly $\mathrm{HBeAg-seroconversion} \mathrm{rate} \mathrm{is} \mathrm{very} \mathrm{high}$ $(14-16 \%)$ and only $10 \%$ are estimated to remain $\mathrm{HBeAg}$-positive when reaching puberty ${ }^{29}$. As a result, most adults with a chronic HBV infection in the region are HBeAg-negative, generally with a low or undetectable HBV VL and no signs of liver inflammation ${ }^{30}$. Early 
seroconversion is potentially explained by the HBV genotype distribution in SSA, as the median age of $\mathrm{HBe}$ seroconversion has been shown to be much lower in persons infected with genotypes A, E and D compared to genotype $C^{31}$. Ten to $20 \%$ of patients in the HBeAgnegative phase will experience viral rebound and exacerbation of hepatitis after years of quiescence, whereas a small fraction of them will even re-convert to $\mathrm{HBeAg-positive} \mathrm{chronic}$ hepatitis $^{32}$. Importantly, individuals with $\mathrm{HBeAg-negative} \mathrm{chronic} \mathrm{hepatitis} \mathrm{may} \mathrm{again}$ experience progression to cirrhosis and develop $\mathrm{HCC}^{33}$. Finally, HBsAg loss (HBV functional cure) occurs at a rate of $0.5-2 \% / y e a r$ in those infected during adolescence but at a lower rate (0.1-1\%/year) in those who acquired chronic HBV infection in early life ${ }^{34}$.

Figure 3: Natural history of chronic HBV infection (new and old nomenclature refer to terms used in the EASL 2017 Clinical Practice Guidelines on the management of hepatitis B virus infection ${ }^{35}$ )

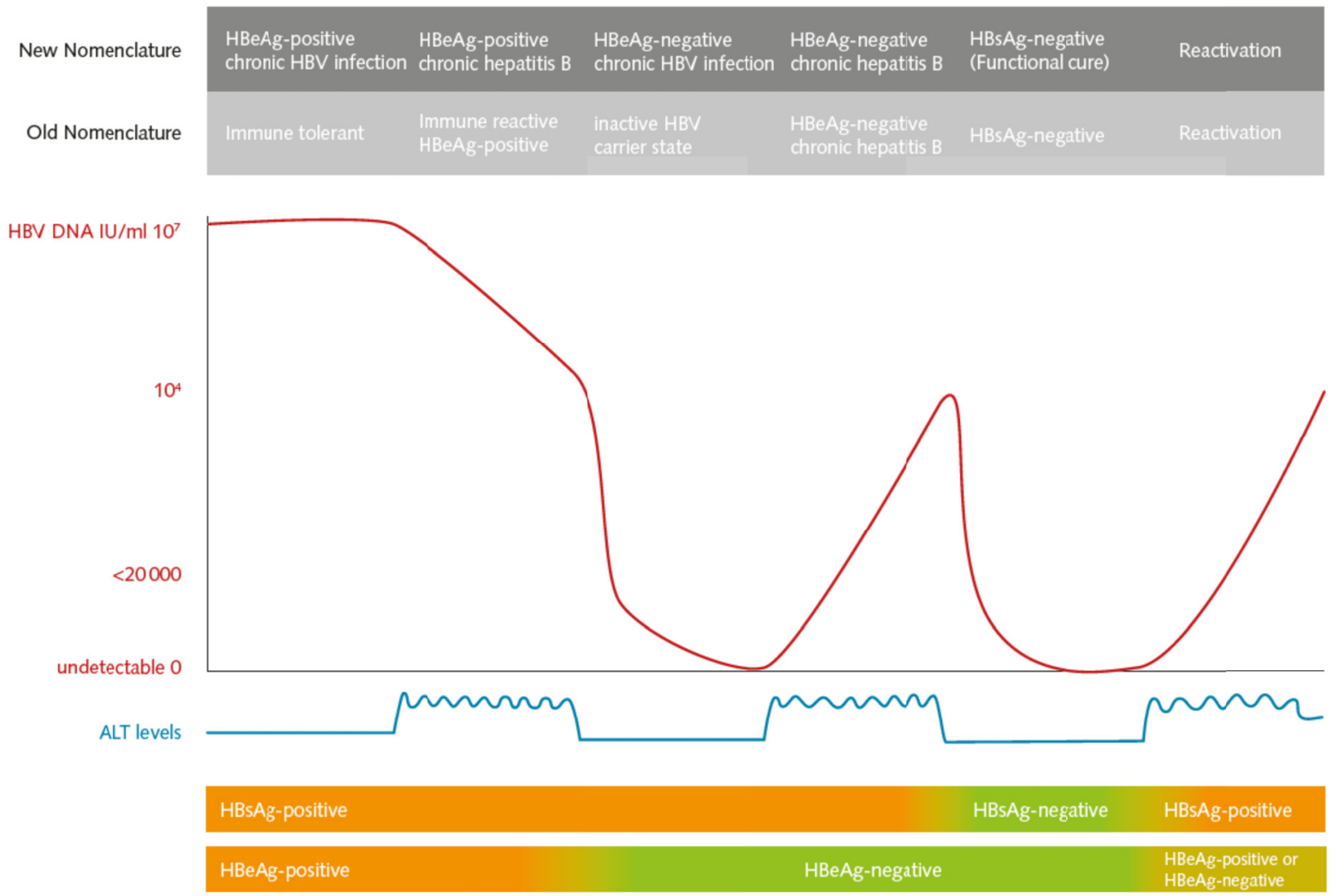

\subsection{Hepatitis B testing: Good assays but low uptake}

Since the prevalence of chronic HBV infection is above $5 \%$ in SSA, WHO recommends HBsAg testing of the general population using a single, quality-assured serological test (either a laboratory-based immunoassay or a rapid diagnostic test) ${ }^{36}$. In West Africa, largescale testing of the general population and treatment of eligible individuals was shown to be cost-effective in a mathematical modeling study, even down to a prevalence below $2 \%{ }^{37}$. 
Based on results from a community outreach program in the Gambia, the PROLIFICA (Prevention of Liver Fibrosis and Liver Cancer in Africa) study team showed that such a screen-and-treat strategy was feasible in $\mathrm{SSA}^{38}$. However, uptake of HBV testing outside of clinical and epidemiological studies has remained dramatically low in the region with less than $1 \%$ of HBV infections being currently diagnosed in Africa ${ }^{3}$. Even in clinical settings such as HIV outpatient clinics, where patients are linked to care, HBV testing rates remain very low. In a study of over 60,000 HIV-infected individuals from fifteen primary care clinics in Lusaka, Zambia, we showed that the uptake of HBV screening was slow and very different across clinical settings ${ }^{39}$. Furthermore, through the review of over 3,500 clinical charts from HIV clinics in ten African countries, large differences in HBV screening uptake patterns across settings were observed, and the overall number of patients tested remained below $25 \%{ }^{40}$. These results are driven by financial constraints, as in many settings patients have to pay for diagnostic tests, but also by the lack of awareness and knowledge on HBV infection among health care workers. In Senegal, only $24 \%$ of physicians are aware of the existence of rapid diagnostic tests for detecting HBV infection ${ }^{6}$.

The availability of rapid tests with decent diagnostic accuracy should help improve HBV diagnosis rates in the near future. For instance, the widely available point-of-care Determine ${ }^{\circledR}$ HBsAg test (Alere, USA) had an overall sensitivity of $87.9 \%$ in HIV-infected individuals in Zambia, when compared to the standard laboratory-based serological test, in line with results from a large, recently published meta-analysis ${ }^{41,42}$. Importantly, sensitivity was $95.9 \%$ among patients with a replicating HBV and $100 \%$ among those with a high HBV VL. Despite the fear that viral escape mutations might lead to false-negative HBsAg screening results, a study among HBV-infected individuals in Switzerland showed that specific resistance mutations associated with vaccine escape mutations did not affect the sensitivity of the test ${ }^{43}$

In order to significantly improve HBV testing rates in resource-limited countries, WHO recommends improving health-facility-based testing by systematically integrating HBV screening in established services such as antenatal and HIV outpatient clinics. In addition, innovative strategies to reach populations not linked to health-care, including vulnerable populations, will have to be adopted. Learning from previous experiences in communitybased HIV testing will be key to rapidly adapt and implement HBV-targeted testing interventions in SSA. For instance, in a large meta-analysis assessing the feasibility of community-based HIV testing strategies, Suthar et al. reported high rates of uptake and acceptability of interventions such as door-to-door, workplace or school-based testing, which could also be used to test for HBV infection ${ }^{44}$. As with HIV testing, specific interventions will have to be established for HBV testing in key populations. 


\section{Assessing HBV activity and degree of liver disease}

The initial evaluation of a subject with chronic HBV infection should include a complete history, a physical examination, the evaluation of blood markers (transaminases, HBV serological tests and VL), and the assessment of liver fibrosis/cirrhosis. Initiation of antiviral therapy is guided by the results of these parameters. A single determination of HBV replication and disease activity markers is generally insufficient to allow an immediate classification into one of the phases of disease (Figure 3 ). Serial measurements of serum HBsAg, HBV DNA and alanine aminotransferase (ALT) levels as well as liver fibrosis stage are required in most instances. Despite a complete assessment, some subjects fall into a grey area and management needs to be individualized. Additional routine investigations include liver ultrasonography for the periodic screening for HCC, and endoscopy for detecting esophageal varices in persons with cirrhosis.

\subsection{Liver inflammation}

Aminotransferase levels, which indicate the presence of liver inflammation, fluctuate over time, justifying the need for serial measurements for disease monitoring. Usually, ALT concentrations are higher than aspartate aminotransferase (AST) levels in chronic HBV infection, but with disease progression to cirrhosis, the AST/ALT ratio may be reversed. As transaminase measurements are widely available, including in primary care clinics across SSA, they are an essential part of determining disease activity in low-income countries and are included in all treatment guidelines.

\subsection{HBV replication}

In SSA, few facilities have the laboratory capacity for measuring HBV VL ${ }^{45}$. The price of commercial assays is high (generally over 50 USD per test), and their use requires specific infrastructure and skilled technicians. Serum HBV DNA concentrations assessed by real-time quantitative polymerase chain reaction is a strong predictor of the risk of developing HCC, and the value is used in the algorithm for deciding who to treat and how to monitor patients ${ }^{45}$. However, since it is measured very rarely in clinical routine in SSA, there is an urgent need for alternative ways to assess HBV replication. For instance, loop-mediated isothermal amplification (LAMP) is a promising new technology, which may soon allow the measurement of HBV VL at reduced cost and in very little time ${ }^{46}$. Furthermore, the measurement of HBV VL from dried blood spots has recently been validated in Zambia and will allow rural clinics in SSA to ship samples to reference labs without facing the need to secure the cold chain ${ }^{47}$. Few studies have assessed the proportion of treatment-naïve HBV- 
infected individuals with high replication in SSA. For example, Aberra et al. showed that in Ethiopia, only a small proportion (22\%) of patients with chronic HBV infection had a VL $>20,000 \mathrm{IU} / \mathrm{ml}$, contrasting with results from a cohort of HIV/HBV-coinfected individuals starting antiretroviral therapy (ART) in Mozambique and Zambia, where $50 \%$ of the participants had an HBV VL $>20,000 \mathrm{IU} / \mathrm{ml}^{48,49}$. Interestingly, in the latter study, all HBeAgpositive patients had a high HBV VL, in line with observations made previously in Malawi ${ }^{50}$. This finding suggests that, in settings with limited access to HBV VL assays, a positive $\mathrm{HBeAg}$ test could be used as a proxy for a high $\mathrm{HBV} \mathrm{VL}^{50}$.

\subsection{Fibrosis assessment}

Liver biopsy, the gold standard for assessing liver fibrosis, is not widely used in low-income countries as it involves high costs and dedicated infrastructure, relies on the skills of trained clinicians and pathologists, and carries the risk of complications and discomfort to the patient. Several non-invasive tests based on serum indices (AST-to-platelet ratio index [APRI], FIB-4) or ultrasound principles (transient elastography [TE]) have been developed to replace liver biopsy for the assessment of liver disease stage. TE is currently the best alternative to liver biopsy, as it has showed excellent accuracy for quantifying liver fibrosis and cirrhosis in HBV-infected patients ${ }^{36,51-53}$, and can be used in outpatient or community settings by non-specialist health-care staff. Unfortunately, the cost of the TE device has limited its widespread use in resource-limited settings. If TE is unavailable, WHO recommends the use of the APRI score, which is based on the measurement of laboratory tests readily available in resource-limited settings ${ }^{54}$. Although APRI has a high specificity for the detection of liver cirrhosis, its low sensitivity for detecting significant fibrosis and cirrhosis at the specified cut-off ranges causes many patients with advanced fibrosis and cirrhosis to be missed ${ }^{55}$.

Although the presence of liver cirrhosis is a clear indication for immediate HBV therapy, few studies have reported on the degree of liver fibrosis in treatment-naïve HBV-infected individuals in SSA to date. In addition, study design, sample size and methods to assess liver fibrosis are highly variable across publications. Figure 4 shows the proportion of HBVinfected individuals with significant fibrosis or cirrhosis from a systematic review of studies that have assessed these parameters in SSA. Six studies including 1,089 patients reported data on fibrosis/cirrhosis in HBV-moninfected patients and 10 studies including 629 patients among HIV/HBV-coinfected patients. Fibrosis assessment was performed with APRI in eight studies, TE in five studies, and FIB-4 and Fibrotest each in one study. The prevalence of liver cirrhosis ranged from $0.3 \%$ to $17 \%$ in HBV-monoinfected and from $4 \%$ to $14 \%$ in HIV/HBVcoinfected individuals. In HBV-monoinfected patients, the proportion with significant fibrosis varied between $4 \%$ and $30 \%$, whereas in HIV/HBV-coinfected ones, estimates seemed to be 
higher, with a range between $12 \%$ and $46 \%$. Heterogeneity in results probably reflect differences between the settings in which the studies were performed. For instance, Aberra et al. found a very high prevalence of liver cirrhosis (17.2\%) among HBV-moninfected individuals in a hospital-based cohort, whereas Shimakawa et al. found a prevalence of cirrhosis $<1 \%$ in a rural community-based cohort, which is probably more representative of the general population ${ }^{30,55}$.

Figure 4: Proportion of HBV-infected individuals with significant fibrosis and cirrhosis, by HIV-infection status (systematic review of the literature performed on $27^{\text {th }}$ November 2017)

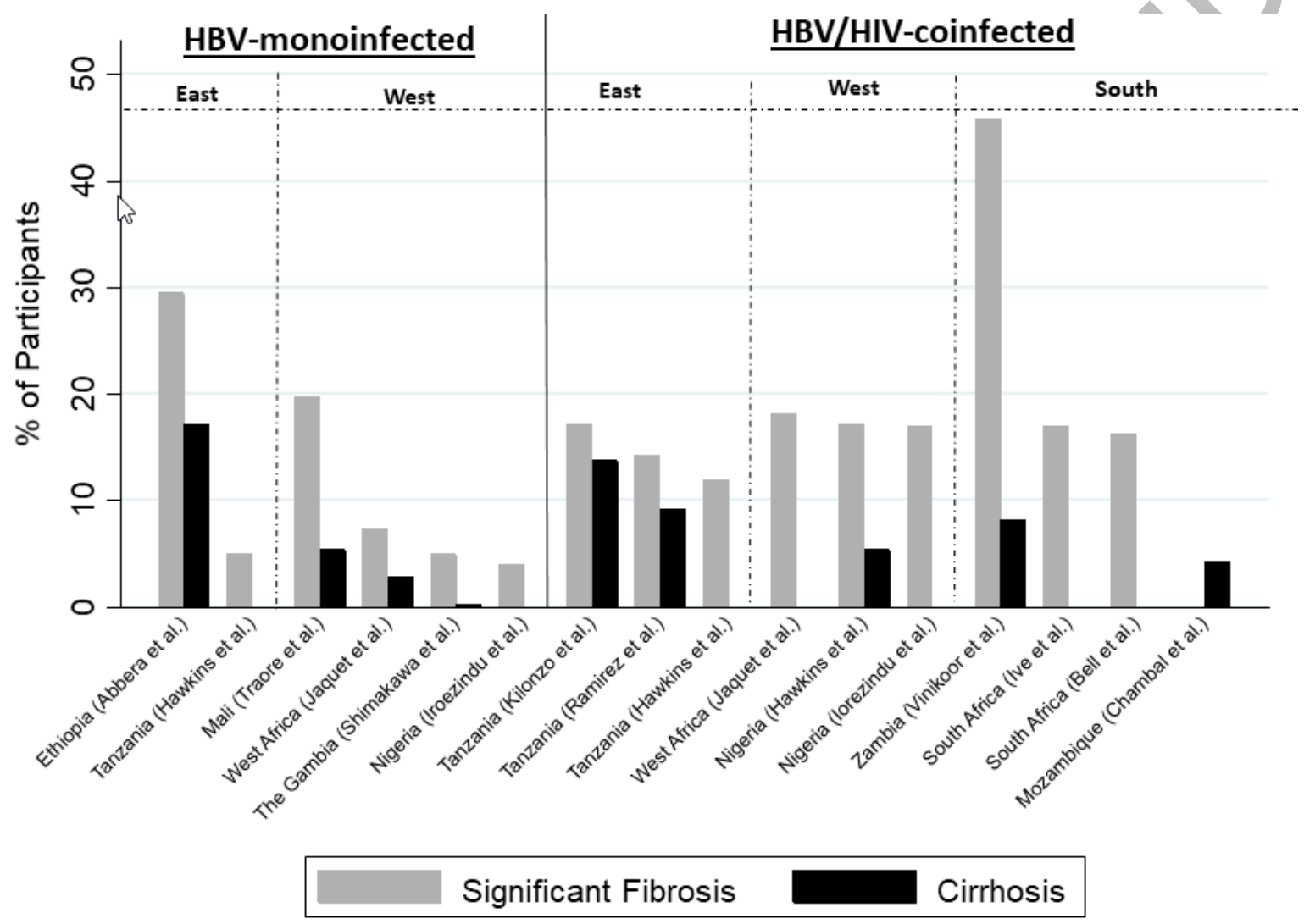

\section{Treatment eligibility, access and outcomes}

The goal of HBV therapy is to improve survival and quality of life by preventing the progression of liver disease and, consequently, the development of HCC. Furthermore, antiviral therapy helps prevent HBV reactivation and mother-to-child transmission, as well as to treat HBV-associated extrahepatic manifestations. The decision to initiate antiviral therapy is usually based on the combined assessment of HBV replication (HBV VL), inflammation $(A L T)$, and stage of liver fibrosis. Several international guidelines, including by WHO, give recommendations on HBV management, but the optimal timing and indications for treatment are still debated ${ }^{35,56,57}$. In general, treatment is targeted at persons with chronic HBV infection who are at high risk of disease progression and HCC: those with moderate or severe liver 
inflammation, and/or fibrosis and high viral replication. The benefits of treatment of HBVinfected individuals with mild inflammation or fibrosis are uncertain. As only very limited longterm data are available from SSA, it is unknown if the impact of antiviral therapy on disease progression is similar compared to other regions.

\subsection{Treatment indication}

WHO recently issued HBV treatment recommendations based on other international guidelines, but including algorithms for resource-limited settings without access to all necessary diagnostic tools ${ }^{5}$. Currently, it is recommended to treat all adults, adolescents and children with chronic HBV infection and evidence of cirrhosis (based on clinical signs, TE or APRI score), regardless of ALT levels, HBeAg status or HBV VL. Treatment is also recommended for non-cirrhotic adults with chronic HBV infection aged more than 30 years with persistently abnormal ALT levels and evidence of high-level HBV replication (HBV DNA $>20,000 \mathrm{IU} / \mathrm{mL}$ ). Where HBV VL testing is unavailable, treatment may be considered based on persistently abnormal ALT levels alone. Furthermore, all HIV/HBV-coinfected individuals should receive TDF-containing ART, independent of CD4 count or stage of liver disease ${ }^{58}$. Continued monitoring is necessary in all persons with a chronic HBV infection, particularly if they do not yet meet the criteria for initiating antiviral therapy.

One of the main research gaps in the field of HBV care in SSA is the lack of prospective cohort data on treatment eligibility. As shown in Figure 4, only three published studies assessed the proportion of HBV-monoinfected patients with cirrhosis using TE, including a total of 677 individuals. In community-based studies from West Africa, between $2 \%$ and $10 \%$ of patients were eligible for antiviral therapy according to WHO guidelines ${ }^{38,59}$. In contrast, a hospital-based study from Ethiopia found a much higher proportion, with approximately one quarter of patients having an indication to start treatment ${ }^{55}$. Importantly, the study by Jaquet et al. highlights the need for prospective studies of patients without an immediate indication for therapy as nearly $50 \%$ of the participants had some degree of HBV-related disease activity, which warranted follow-up measurements ${ }^{59}$. In summary, there is an alarming paucity of data available on HBV treatment needs in SSA, which is essential information for the planning of resource allocation to fight the global HBV epidemic.

\subsection{Treatment options and outcomes}

In high-income countries, two distinct HBV treatment modalities have been commonly used: 1) pegylated interferon (pegIFN), which, given as weekly injections for $\geq 12$ months, has the potential to achieve HBV functional cure, but is expensive and leads to severe side-effects, and 2) nucleoside analogues (NUC), which are well tolerated but require lifelong therapy in most patients. TDF is currently the NUC of choice as it is very effective in achieving an 
undetectable HBV VL as well as the normalization of ALT levels, and has few side-effects. Unlike with other NUC such as lamivudine or entecavir, the development of HBV resistance to TDF has not yet been documented in clinical studies. Lately, tenofovir alafenamide fumarate (TAF), a prodrug of tenofovir that enables enhanced delivery of the parent nucleotide and its active diphosphate metabolite into lymphoid cells and hepatocytes, was shown to be non-inferior to TDF for the treatment of HBV infection, while being associated with a lower risk of developing nephrotoxicity or osteopenia ${ }^{60}$. However, TAF is generally not yet available in SSA.

Suppression of HBV replication with TDF reduces the degree of chronic liver inflammation and leads to the regression of liver fibrosis $^{61}$. In SSA, several observational studies have shown excellent rates of HBV suppression in cohorts of HIVinfected individuals, and rates of HBsAg loss appear to be higher than among HBVmonoinfected patients ${ }^{62,63}$. Furthermore, a significant proportion of HIV/HBVcoinfected individuals started on TDF-containing ART in Zambia showed a regression in liver fibrosis stage after one year of

\section{Box 1. Access to HBV therapy in Senegal}

After having been confronted with difficulties in the care of HBV-infected individuals for many years, members of the Senegalese Society of Gastroenterology (SOSEGH) approached the National AIDS program to discuss a potential collaboration in 2006. The latter offered to make lamivudine available for HBV-infected individuals in need of treatment in Dakar, the capital city. As TDF became available for HIV-infected individuals in 2010, HBV-infected persons also received access to this new drug, again through the National AIDS program. However, stock-outs were common, so that many physicians had to switch patients back to lamivudine to guarantee the continuity of their treatment. After several years of discussion with the SOSEGH, the Senegalese Ministry of Health agreed to provide TDF for the treatment of HBV infection in early 2017. Generic TDF is now available for HBV-infected patients at a reduced price of approximately 10 USD per month at four main hospitals in Dakar. Stock-outs still occur, but their frequency could be reduced if generic drugs were made available to public

therapy ${ }^{62}$. Finally, TDF is also beneficial in reducing the incidence of HCC among HBVinfected populations ${ }^{64}$, but similar studies have not yet been performed in African settings, where other HCC risk factors such as aflatoxin and alcohol consumption are prevalent.

\subsection{Treatment access}

Few African countries have developed national treatment strategies for the management of chronic HBV infection ${ }^{65}$. Although TDF is now part of first-line ART for HIV infection in most resource-limited countries, access to this drug for HBV-monoinfected individuals has remained severely restricted. However, several countries in SSA have recently made important steps towards the access to generic TDF for all HBV-infected individuals in need of therapy. Often, the determination and commitment of dedicated physicians and members of 
the civil society were crucial in this process, as exemplified by the situation in Senegal (Box 1).

\subsection{Complications and co-morbidities}

The main complications of chronic HBV infection are the development of liver cirrhosis and HCC. According to the few cross-sectional studies available from SSA, up to $17 \%$ of HBVinfected patients present for care with liver cirrhosis (Figure 4). However, data on the risk of liver decompensation in these patients are scarce. In a recent clinical survey of patients hospitalized with signs of liver decompensation in Uganda, one quarter had a chronic HBV infection and the main complication of cirrhosis was the development of ascites ${ }^{66}$.

Over $50 \%$ of cases of HCC can be attributed to HBV infection in SSA, whereas $22 \%$ of them are HBV-related in Europe ${ }^{67}$. Large observational studies from Asia showed that HBV-related $\mathrm{HCC}$ incidence was strongly associated with the presence of cirrhosis, high HBV VL, genotype, and HBeAg-seropositivity ${ }^{68-70}$. However, data on HCC from SSA are poor and generally limited to case-control studies ${ }^{71,72}$. In one of few longitudinal studies assessing the incidence of HBV-related HCC in SSA, Shimakawa et al. reported an incidence of 56 cases per 100,000 person-years among 405 chronic carriers, with a median follow-up of 28 years in the Gambia $^{30}$.

Most studies comparing the risk of HCC between patients on potent NUC and matched untreated controls found a significant risk reduction with NUC therapy but the overall risk was not eliminated ${ }^{73}$. Unlike the natural history of HCV-related HCC, which is driven by liver cirrhosis, HBV-related HCC also develops in non-cirrhotics, which makes surveillance strategies more complex ${ }^{74}$. Because of the potential synergistic carcinogenic interaction between aflatoxin B1, a fungal toxin which often contaminates foodstuffs in SSA, and HBV, patients of African origin are thought to be more likely to develop HCC at a young age ${ }^{75}$. However, this observation has been much debated and could be explained by the specific age distribution of the general population ${ }^{76}$. The systematic screening for HCC aims at reducing mortality by diagnosing small lesions that are potentially curable by resection, ablation or transplantation. However, he need for HCC surveillance in non-cirrhotic patients successfully treated with TDF is debated. Thus, HBV-related HCC surveillance programs in SSA would benefit from a risk score to help clinicians target groups of individuals most at risk of developing an HCC. Unfortunately, management capacity of HBV-related complications are currently limited in SSA, except from a few large teaching hospitals.

Besides the synergistic effect of HIV infection on the development of HBV-related liver complications (see above), hepatitis delta virus (HDV) infection is an additional HCC risk factor. In a recent study from the Swiss HIV Cohort Study, HIV/HBV/HDV-coinfected individuals were nine times more likely to develop an HCC compared to their HDV-uninfected 
counterparts $^{77}$. Currently, there are still uncertainties about the epidemiology of HDV infection in SSA. According to a recently published meta-analysis, the proportion of HBV-infected individuals with an HDV coinfection ranges from $0.1 \%$ in southern Africa to $25.6 \%$ in central Africa $^{78}$. Thus, estimates vary widely across regions and sometimes even within countries, which underlines the need to better understand the contribution of HDV infection to the burden of HCC in SSA.

\section{Conclusion}

The availability of good diagnostic tests and potent antiviral therapy, as well as the fastgrowing pipeline of drugs expected to help achieve HBV functional cure, have led WHO and other stakeholders to set HBV elimination by 2030 as a major global health target. However, current levels of dedication and funding to fill the many related research gaps are insufficient to reach this goal. In particular, knowledge about the main epidemiological and clinical determinants of HBV infection in SSA is still very limited. In order to inform the design and implementation of public health strategies, there is an urgent need for dedicated, long-term cohort studies aiming at understanding the natural history and treatment outcomes of HBVinfected individuals in the region.

HBV-related mortality will continue to increase if measures to improve its management in resource-limited countries are not taken rapidly. International recommendations now clearly stipulate the need for HBV screening of the general population in SSA, which should be facilitated by the widespread availability of rapid HBsAg tests. Tools to assess individual treatment eligibility at low cost, including the evaluation of liver fibrosis and viral replication need to be developed and validated in the region. Although access to TDF for the treatment of chronic hepatitis B seems to improve slowly, efforts from health authorities and policy makers will be crucial to allow proper management of HBV infection for all people infected and, eventually, achieve the global elimination of the infection.

\section{Expert commentary}

Eliminating viral hepatitis as a major public health problem by 2030 must be our common goal. According to WHO, HBV testing has to increase from less than $1 \%$ to $80 \%$ in subSaharan Africa (SSA) if that ambitious target is to be met. This is very challenging, considering that current rates of routine HBV testing are very poor, even in health-care settings such as HIV clinics, where HBV screening has been recommended for years. Thus, major efforts are necessary to increase HBV testing uptake in established clinical structures, and to evaluate novel, acceptable community-based testing strategies. Recent experiences in the field of HIV testing in SSA should be a source of inspiration for the design of such programs. As low rates of HBV testing are mainly due to insufficient awareness and financial 
resources, existing point-of-care tests with good diagnostic accuracy are to be made widely available across SSA.

Obviously, diagnosing HBV infections is only the first step in the continuum of HBV care. The measurement of HBV viral load (VL) and the assessment of the degree of liver fibrosis are cornerstones of HBV management. Currently, access to optimal diagnostic tools, such as HBV amplification assays and transient elastography, is limited by their high costs. Although several non-invasive serological scores for the assessment of liver fibrosis have been validated in different settings, they generally have a poor diagnostic accuracy. For instance, the test currently recommended by WHO, the APRI score, fails to detect the majority of cases of liver cirrhosis. Therefore, advances in the development of affordable point-of-care HBV VL assays as well as tools to assess liver fibrosis are urgently needed.

Fortunately, access to generic tenofovir, the mainstay of HBV-therapy, is becoming a reality in an increasing number of clinical settings in low-income countries. Although the proportion of individuals with an indication for therapy in SSA remains to be determined, there is emerging evidence on the benefits of antiviral therapy on HBV-related clinical outcomes, including the improvement of liver fibrosis and the reduction of the incidence of hepatocellular carcinoma. However, the lack of long-term prospective cohorts dedicated to the study of HBV-related outcomes in SSA represents one of the major current research gaps. Considering the number of antiviral drugs with the potential for achieving the functional cure of HBV infection currently in the pipeline, a better understanding of the main determinants of HBV infection and its complications is urgent. Therefore, increased funding for HBV research in SSA needs to be advocated for.

In summary, the tools needed to diagnose and evaluate HBV infection, as well as antiviral therapy to suppress its replication are well established. However, most current diagnostic methods are either too expensive to be rolled out at a large scale in SSA, not validated, or not accurate enough. As the functional cure of HBV infection is becoming a realistic goal with future treatment modalities, dedicated research cohorts are needed to inform future needs of African healthcare programs so that strategies for the elimination of HBV infection can be tailored to specific settings.

\section{Five-year view}

In order to achieve HBV elimination, the natural history of HBV and treatment outcomes will have to be evaluated in dedicated research cohorts in SSA, which will be established in parallel to the upscale of HBV care interventions. Both the scientific community as well as policy makers will depend on findings from such studies to design sound public health interventions. The implementation of specific community-based testing strategies, adapted to the local contexts, will be evaluated and will lead to the establishment of cost-effective 
screening programs and successful linkage to care of patients diagnosed. Finally, validated point-of-care tests will allow the measurement of HBV VL and adapted fibrosis scores will help clinicians in deciding who should be treated and how patients should be monitored. However, despite the improved access to diagnostic tools and antiviral therapy, the implementation of general HBV testing as well as linkage to and retention in care will remain a challenge, as observed since many years in the fight against HIVIAIDS.

The first phase 2 trials evaluating combinations of newer drugs with the prospect of achieving the functional cure of a majority of HBV-infected individuals will be underway within the next five years. These novel treatment modalities are expected to allow a finite duration of treatment, which will, in the end, also reduce the financial burden of HBV management on national health systems. As the functional cure of HBV infection has been shown to lower the incidence of $\mathrm{HCC}$, it can be expected that the rate of HBV-related complications will also decrease with time.

\section{Key issues}

- HBV infection is the most important cause of liver cirrhosis and hepatocellular carcinoma in sub-Saharan Africa (SSA).

- To achieve HBV elimination as a major public health issue by 2030 , the proportion of individuals tested needs to reach $90 \%$ and those treated $80 \%$.

- Currently, less than $1 \%$ of HBV-infected individuals are diagnosed in SSA and access to antiviral therapy remains limited

- Between $1 \%$ and $17 \%$ of HBV-infected individuals in SSA present with liver cirrhosis, depending on the clinical setting.

- Prospective cohorts aiming at the long-term assessment of treatment eligibility and outcomes, as well as the evaluation of the incidence and risk factors of hepatocellular carcinoma are urgently needed in SSA.

\section{Funding}

G. Wandeler was supported by an Ambizione-PROSPER fellowship from the Swiss National Science Foundation (PZOOP3_177118). The funders had no role in study design, data collection and analysis, decision to publish, or preparation of the manuscript.

\section{Declaration of interest}

The authors have no other relevant affiliations or financial involvement with any organization or entity with a financial interest in or financial conflict with the subject matter or materials discussed in the manuscript apart from those disclosed. Peer reviewers on this manuscript have no relevant financial or other relationships to disclose. 


\section{References}

Papers of special note have been highlighted as:

* of interest

** of considerable interest

1. Global Burden of Disease Cancer C, Fitzmaurice C, Allen C, et al. Global, Regional, and National Cancer Incidence, Mortality, Years of Life Lost, Years Lived With Disability, and Disability-Adjusted Life-years for 32 Cancer Groups, 1990 to 2015: A Systematic Analysis for the Global Burden of Disease Study. JAMA Oncol. 2017;3(4):524-548.

2. WHO guidelines on hepatitis $B$ and $C$ testing. Geneva: World Health Organization; 2017, http://apps.who.int/iris/bitstream/10665/254621/1/9789241549981eng.pdf?ua=1.

3. Schweitzer A, Horn J, Mikolajczyk RT, Krause G, Ott JJ. Estimations of worldwide prevalence of chronic hepatitis $B$ virus infection: a systematic review of data published between 1965 and 2013. Lancet. 2015;386(10003):1546-1555.

4. WHO, Global hepatitis report, 2017. Geneva: World Health Organization, April, 2017. www.who.int/hepatitis/publications/global-hepatitis-report2017/en/

5. WHO Guidelines for the prevention, care and treatment of persons with chronic hepatitis B infection 2015,

http://apps.who.int/iris/bitstream/10665/154590/1/9789241549059 eng.pdf?ua=1 \&ua=1.

***Global recommendations on management of HBV infection

6. Jaquet A, Wandeler G, Tine J, et al. Prevention and Care of Hepatitis B in Senegal; Awareness and Attitudes of Medical Practitioners. Am J Trop Med Hyg. 2017;97(2):389-395.

7. Zanetti AR, Van Damme $P$, Shouval D. The global impact of vaccination against hepatitis B: a historical overview. Vaccine. 2008;26(49):6266-6273.

8. Jacobs B, Mayaud P, Changalucha J, et al. Sexual transmission of hepatitis B in Mwanza, Tanzania. Sex Transm Dis. 1997;24(3):121-126.

9. Dolan K, Wirtz AL, Moazen B, et al. Global burden of HIV, viral hepatitis, and tuberculosis in prisoners and detainees. Lancet. 2016;388(10049):1089-1102.

10. Belo AC. Prevalence of hepatitis B virus markers in surgeons in Lagos, Nigeria. East Afr Med J. 2000;77(5):283-285.

11. Olubuyide IO, Ola SO, Aliyu B, et al. Prevalence and epidemiological characteristics of hepatitis B and C infections among doctors and dentists in Nigeria. East Afr Med J. 1997;74(6):357-361. 
12. Bigna JJ, Amougou MA, Asangbeh SL, et al. Seroprevalence of hepatitis B virus infection in Cameroon: a systematic review and meta-analysis. BMJ Open. 2017;7(6):e015298.

13. Vray M, Debonne JM, Sire JM, et al. Molecular epidemiology of hepatitis B virus in Dakar, Senegal. J Med Virol. 2006;78(3):329-334.

14. Jaquet $A$, Wandeler $G$, Tine J, et al. HIV infection, viral hepatitis and liver fibrosis among prison inmates in West Africa. BMC infectious diseases. 2016;16:249.

15. Diop M, Diouf A, Seck SM, et al. [Prevalence of hepatitis B surface antigen and its associated factors in Senegalese military personnel sent on mission to Darfur]. Pan Afr Med J. 2017;26:154.

16. Jaquet $A$, Wandeler $G$, Nouaman $M$, et al. Alcohol use, viral hepatitis and liver fibrosis among HIV-positive persons in West Africa: a cross-sectional study. J Int AIDS Soc. 2017;19(1):21424.

17. Easterbrook P PL, Gower E, Razavi H, Sabin K, Vickerman P. Global systematic review and metaanalysis of the seroprevalence of HBV and HCV infection in HIVinfected persons. Abstract 254. 8th IAS Conference on Pathogenesis, Treatment and Prevention,19-22 July 2015, Vancouver, Canada.

18. Nikolopoulos GK, Paraskevis D, Hatzitheodorou E, et al. Impact of hepatitis B virus infection on the progression of AIDS and mortality in HIV-infected individuals: a cohort study and meta-analysis. Clin Infect Dis. 2009;48(12):1763-1771.

19. Stabinski L, Reynolds SJ, Ocama $P$, et al. High prevalence of liver fibrosis associated with HIV infection: A study in rural Rakai, Uganda. Antiviral Therapy. 2011;16(3):405411.

20. Edmunds WJ, Medley GF, Nokes DJ, O'Callaghan CJ, Whittle HC, Hall AJ. Epidemiological patterns of hepatitis B virus (HBV) in highly endemic areas. Epidemiol Infect. 1996;117(2):313-325.

21. Dumpis $U$, Holmes EC, Mendy M, et al. Transmission of hepatitis B virus infection in Gambian families revealed by phylogenetic analysis. J Hepatol. 2001;35(1):99-104.

22. Merican I, Guan R, Amarapuka D, et al. Chronic hepatitis B virus infection in Asian countries. J Gastroenterol Hepatol. 2000;15(12):1356-1361.

23. Acquaye JK, Mingle JA. Hepatitis B viral markers in Ghanaian pregnant women. West Afr J Med. 1994;13(3):134-137.

24. Menendez C, Sanchez-Tapias JM, Kahigwa E, et al. Prevalence and mother-to-infant transmission of hepatitis viruses B, C, and E in Southern Tanzania. J Med Virol. $1999 ; 58(3): 215-220$. 
25. Matthews PC, Beloukas A, Malik A, et al. Prevalence and Characteristics of Hepatitis $B$ Virus (HBV) Coinfection among HIV-Positive Women in South Africa and Botswana. PLoS One. 2015;10(7):e0134037.

26. Matthews PC, Geretti AM, Goulder PJ, Klenerman P. Epidemiology and impact of HIV coinfection with hepatitis B and hepatitis $C$ viruses in Sub-Saharan Africa. J Clin Virol. 2014;61(1):20-33.

27. Eke CB, Ogbodo SO, Ukoha OM, et al. Seroprevalence and Risk Factors of Hepatitis $B$ Virus Infection among Adolescents in Enugu, Nigeria. J Trop Pediatr. 2015;61(6):407-413.

28. Abebe A, Nokes DJ, Dejene A, Enquselassie F, Messele T, Cutts FT. Seroepidemiology of hepatitis B virus in Addis Ababa, Ethiopia: transmission patterns and vaccine control. Epidemiol Infect. 2003;131(1):757-770.

29. Bile K, Abdirahman M, Mohamud O, et al. Late seroconversion to hepatitis B in a Somali village indicates the important role of venereal transmission. J Trop Med Hyg. 1991;94(6):367-373.

30. Iorio R, Giannattasio A, Cirillo F, L DA, Vegnente A. Long-term outcome in children with chronic hepatitis B: a 24-year observation period. Clin Infect Dis. 2007;45(8):943949.

31. Shimakawa Y, Lemoine M, Njai HF, et al. Natural history of chronic HBV infection in West Africa: a longitudinal population-based study from The Gambia. Gut. 2016;65(12):2007-2016.

*** Most comprehensive proespective study on HBV natural history in subSaharan Africa

32. Livingston SE, Simonetti JP, Bulkow LR, et al. Clearance of hepatitis B e antigen in patients with chronic hepatitis B and genotypes A, B, C, D, and F. Gastroenterology. 2007;133(5):1452-1457.

33. Lok AS, McMahon BJ. Chronic hepatitis B. Hepatology. 2007;45(2):507-539.

34. Hadziyannis SJ, Vassilopoulos D. Hepatitis B e antigen-negative chronic hepatitis B. Hepatology. 2001;34(4 Pt 1):617-624.

35. Liaw YF, Sheen IS, Chen TJ, Chu CM, Pao CC. Incidence, determinants and significance of delayed clearance of serum HBsAg in chronic hepatitis $B$ virus infection: a prospective study. Hepatology. 1991;13(4):627-631.

36. European Association for the Study of the Liver. Electronic address eee, European Association for the Study of the L. EASL 2017 Clinical Practice Guidelines on the management of hepatitis B virus infection. J Hepatol. 2017;67(2):370-398. 
37. Miailhes $P$, Pradat $P$, Chevallier $M$, et al. Proficiency of transient elastography compared to liver biopsy for the assessment of fibrosis in HIV/HBV-coinfected patients. Journal of viral hepatitis. 2011;18(1):61-69.

38. Nayagam S, Thursz M, Sicuri E, et al. Requirements for global elimination of hepatitis B: a modelling study. The Lancet. Infectious diseases. 2016;16(12):1399-1408.

39. Lemoine M, Shimakawa Y, Njie R, et al. Acceptability and feasibility of a screen-andtreat programme for hepatitis $B$ virus infection in The Gambia: the Prevention of Liver Fibrosis and Cancer in Africa (PROLIFICA) study. Lancet Glob Health. 2016;4(8):e559-567.

40. Vinikoor MJ, Musukuma K, Munamunungu V, et al. Implementation of routine screening for chronic hepatitis B virus co-infection by HIV clinics in Lusaka, Zambia. Journal of viral hepatitis. 2015;22(10):858-860.

41. Coffie PA, Tchounga BK, Bado $G$, et al. Prevalence of hepatitis $B$ and delta according to HIV-type: a multi-country cross-sectional survey in West Africa. BMC infectious diseases. 2017;17(1):466.

42. Chisenga CC, Musukuma K, Chilengi R, et al. Field performance of the Determine HBsAg point-of-care test for diagnosis of hepatitis B virus co-infection among HIV patients in Zambia. Journal of clinical virology : the official publication of the Pan American Society for Clinical Virology. 2018;98:5-7.

43. Amini A, Varsaneux $O$, Kelly $H$, et al. Diagnostic accuracy of tests to detect hepatitis $B$ surface antigen: a systematic review of the literature and meta-analysis. BMC infectious diseases. 2017;17(Suppl 1):698.

44. Hirzel C, Pfister S, Gorgievski-Hrisoho M, Wandeler G, Zuercher S. Performance of HBsAg point-of-care tests for detection of diagnostic escape-variants in clinical samples. J Clin Virol. 2015;69:33-35.

45. Suthar AB, Ford N, Bachanas PJ, et al. Towards universal voluntary HIV testing and counselling: a systematic review and meta-analysis of community-based approaches. PLoS medicine. 2013;10(8):e1001496.

46. Chen CJ, lloeje UH, Yang HI. Long-term outcomes in hepatitis B: the REVEAL-HBV study. Clin Liver Dis. 2007;11(4):797-816, viii.

47. Nyan DC, Ulitzky LE, Cehan N, et al. Rapid detection of hepatitis B virus in blood plasma by a specific and sensitive loop-mediated isothermal amplification assay. Clin Infect Dis. 2014;59(1):16-23.

48. Vinikoor MJ, Zurcher S, Musukuma K, et al. Hepatitis B viral load in dried blood spots: A validation study in Zambia. J Clin Virol. 2015;72:20-24. 
49. Aberra $\mathrm{H}$, Desalegn $\mathrm{H}$, Berhe $\mathrm{N}$, et al. Early experiences from one of the first treatment programs for chronic hepatitis B in sub-Saharan Africa. BMC infectious diseases. 2017;17(1):438.

50. Wandeler G, Musukuma K, Zurcher S, et al. Hepatitis B Infection, Viral Load and Resistance in HIV-Infected Patients in Mozambique and Zambia. PloS one. 2016;11(3):e0152043.

51. Aoudjane S, Chaponda M, Gonzalez Del Castillo AA, et al. Hepatitis B Virus Subgenotype A1 Infection Is Characterized by High Replication Levels and Rapid Emergence of Drug Resistance in HIV-Positive Adults Receiving First-line Antiretroviral Therapy in Malawi. Clin Infect Dis. 2014;59(11):1618-1626.

52. Chon YE, Choi EH, Song KJ, et al. Performance of transient elastography for the staging of liver fibrosis in patients with chronic hepatitis B: a meta-analysis. PloS one. 2012;7(9):e44930.

53. Bonnard P, Sombie R, Lescure FX, et al. Comparison of elastography, serum marker scores, and histology for the assessment of liver fibrosis in hepatitis B virus (HBV)infected patients in Burkina Faso. Am J Trop Med Hyg. 2010;82(3):454-458.

54. Marcellin P, Ziol M, Bedossa $P$, et al. Non-invasive assessment of liver fibrosis by stiffness measurement in patients with chronic hepatitis B. Liver Int. 2009;29(2):242247.

55. Wai CT, Greenson JK, Fontana RJ, et al. A simple noninvasive index can predict both significant fibrosis and cirrhosis in patients with chronic hepatitis C. Hepatology. 2003;38(2):518-526.

56. Desalegn H, Aberra H, Berhe N, Gundersen SG, Johannessen A. Are non-invasive fibrosis markers for chronic hepatitis B reliable in sub-Saharan Africa? Liver Int. 2017;37(10):1461-1467.

57. Koh C, Zhao X, Samala N, Sakiani S, Liang TJ, Talwalkar JA. AASLD clinical practice guidelines: a critical review of scientific evidence and evolving recommendations. Hepatology. 2013;58(6):2142-2152.

58. Sarin SK, Kumar M, Lau GK, et al. Asian-Pacific clinical practice guidelines on the management of hepatitis B: a 2015 update. Hepatol Int. 2016;10(1):1-98.

59. Rusthoven CG, Lanning RM, Jones BL, et al. Metastatic nasopharyngeal carcinoma: Patterns of care and survival for patients receiving chemotherapy with and without local radiotherapy. Radiotherapy and oncology : journal of the European Society for Therapeutic Radiology and Oncology. 2017;124(1):139-146.

60. Jaquet A, Nouaman M, Tine J, et al. Hepatitis B treatment eligibility in West Africa: Uncertainties and need for prospective cohort studies. Liver Int. 2017;37(8):11161121. 
61. Agarwal K, Fung SK, Nguyen TT, et al. Twenty-eight day safety, antiviral activity, and pharmacokinetics of tenofovir alafenamide for treatment of chronic hepatitis $B$ infection. J Hepatol. 2015;62(3):533-540.

62. Marcellin P, Gane E, Buti M, et al. Regression of cirrhosis during treatment with tenofovir disoproxil fumarate for chronic hepatitis B: a 5-year open-label followup study. Lancet. 2013;381(9865):468-475.

**Landmark paper on the impact of antiviral therapy on HBV-related liver fibrosis

63. Vinikoor MJ, Sinkala E, Chilengi R, et al. Impact of Antiretroviral Therapy on Liver Fibrosis Among Human Immunodeficiency Virus-Infected Adults With and Without HBV Coinfection in Zambia. Clin Infect Dis. 2017;64(10):1343-1349. **largest study to assess liver fibrosis prospectively among HIV/HBVcoinfected pateitns in sub-Saharan Africa

64. Stockdale AJ, Phillips RO, Beloukas A, et al. Liver Fibrosis by Transient Elastography and Virologic Outcomes After Introduction of Tenofovir in Lamivudine-Experienced Adults With HIV and Hepatitis B Virus Coinfection in Ghana. Clin Infect Dis. 2015;61(6):883-891.

65. Papatheodoridis GV, Idilman R, Dalekos GN, et al. The risk of hepatocellular carcinoma decreases after the first 5 years of entecavir or tenofovir in Caucasians with chronic hepatitis B. Hepatology. 2017;66(5):1444-1453. *Large study showing the impact of antiviral therapy on HBV-related liver cancer

66. Easterbrook $\mathrm{P}$, Sands A, Harmanci H. Challenges and priorities in the management of HIV/HBV and HIV/HCV coinfection in resource-limited settings. Seminars in liver disease. 2012;32(2):147-157.

67. Apica BS, Ocama P, Seremba E, Opio KC, Kagimu MM. Decompensated cirrhosisrelated admissions in a large urban hospital in Uganda: prevalence, clinical and laboratory features and implications for planning patient management. African health sciences. 2013;13(4):927-932.

68. Bosch FX, Ribes J, Diaz M, Cleries R. Primary liver cancer: worldwide incidence and trends. Gastroenterology. 2004;127(5 Suppl 1):S5-S16.

69. Chen CJ, Yang HI, Su J, et al. Risk of hepatocellular carcinoma across a biological gradient of serum hepatitis B virus DNA level. JAMA. 2006;295(1):65-73.

70. Yu MW, Yeh SH, Chen PJ, et al. Hepatitis B virus genotype and DNA level and hepatocellular carcinoma: a prospective study in men. Journal of the National Cancer Institute. 2005;97(4):265-272. 
71. Yang HI, Lu SN, Liaw YF, et al. Hepatitis B e antigen and the risk of hepatocellular carcinoma. The New England journal of medicine. 2002;347(3):168-174.

72. Tanon A, Jaquet A, Ekouevi DK, et al. The spectrum of cancers in West Africa: associations with human immunodeficiency virus. PLoS One. 2012;7(10):e48108.

73. Mendy ME, Welzel T, Lesi OA, et al. Hepatitis B viral load and risk for liver cirrhosis and hepatocellular carcinoma in The Gambia, West Africa. Journal of viral hepatitis. 2010;17(2):115-122.

74. Papatheodoridis GV, Dalekos GN, Yurdaydin C, et al. Incidence and predictors of hepatocellular carcinoma in Caucasian chronic hepatitis $B$ patients receiving entecavir or tenofovir. J Hepatol. 2015;62(2):363-370.

75. Bruix J, Sherman M, Practice Guidelines Committee AAftSoLD. Management of hepatocellular carcinoma. Hepatology. 2005;42(5):1208-1236.

76. Kew MC. Synergistic interaction between aflatoxin B1 and hepatitis B virus in hepatocarcinogenesis. Liver international : official journal of the International Association for the Study of the Liver. 2003;23(6):405-409.

77. Shimakawa Y, Lemoine M. Early age at diagnosis of hepatocellular carcinoma in subSaharan Africa. The lancet. Gastroenterology \& hepatology. 2017;2(6):393.

78. Beguelin C, Moradpour D, Sahli R, et al. Hepatitis delta-associated mortality in HIV/HBV-coinfected patients. J Hepatol. 2017;66(2):297-303.

79. Stockdale AJ, Chaponda M, Beloukas A, et al. Prevalence of hepatitis D virus infection in sub-Saharan Africa: a systematic review and meta-analysis. The Lancet. Global health. 2017;5(10):e992-e1003. 